\title{
Real-Time Cyber-Physical Systems Transatlantic Engineering Curricula Framework
}

\author{
Wojciech Grega \\ Department of Automatics and Biomedical \\ Engineering \\ AGH University of Science and Technology \\ Kraków, Poland \\ wgr@agh.edu
}

\begin{abstract}
${ }^{I}$ Abstract-How to educate future engineers, so that they acquired new skills and competences to become developers of Cyber Physical Systems (CPS)? The paper demonstrates a curriculum framework that was developed and successfully implemented some years ago, as an outcome of two international projects undertaken by a consortium of the European and American universities. The projects had focused on special category of CPS - Real-Time CPS. Examples of laboratory environment supporting education in the field of Real Time CPS are given in the paper.
\end{abstract}

C YBER Physical System is an example of advanced complex technological system interfacing with physical plant and with computing environment running software intensive and most often time critical application. CPS's are often safety-critical. In case of missed time deadlines or component failures CPS may result in violating safety constraints and event life-threatening consequences. We refer to such systems as Real-Time Cyber-Physical Systems (R-TCPS).

Car airbag system is a good example of such system. When a cyber-physical safety system in a car detects a crash, the airbag must inflate in tens of milliseconds to avoid injuries of the driver. The success of the airbag system relies upon the crash sensors working accurately and extremely quickly but also upon algorithms processing in real time. Such a time-critical and safety critical functionality requires specific engineering solutions.

Typically, the challenges of R-TCPS are viewed as separate problems of data measurement, transfer, distributed control, real time operating systems, etc. Domain experts contribute individual components of a design, but they often lack cross-disciplinary knowledge of how these components interact and what impact their behavior may have on other components. As the prevalence and sophistication of cyberphysical systems increases, so does the need for developers with sufficient cross-disciplinary education. In summary, designing of R-TCPS require a multi-disciplinary and

\footnotetext{
${ }^{1}$ The work presented in this paper was supported by the AGH University of Science and Technology, grants number 11.11.120.768, 11.11.120.396 and by the U.S. Department of Education FIPSE grants P116J060005 and P116J080025.
}

\author{
Andrew J. Kornecki \\ Electrical, Computer, Software and System \\ Engineering \\ Embry Riddle Aeronautical University \\ Daytona Beach, FL, USA \\ kornecka@erau.edu
}

integrating knowledge, covering a broad area, from control theory to real-time computing.

The problem addressed in this paper is education: how to educate future developers of R-TCPS that would often work in multinational companies interacting with fellow engineers from different countries?

The following ABET Engineering Criteria Program Educational Outcomes were formulated for accreditation process of CPS education [1]:

- Ability to apply mathematical models of physical systems, cyber systems, and their composition.

- Ability to design and conduct simulations and tests of a cyber-physical system and to analyze the results.

- Ability to apply good engineering practices in the design of a system that mixes cyber and physical components subject to constraints including safety, security, cost, and dependability.

- Ability to function effectively on multi-disciplinary teams spanning cyber and physical domains.

- Ability to identify, formulate and solve engineering problems that have both cyber and physical aspects.

- Understanding of the professional and ethical responsibilities of the design of life- and safety-critical systems.

- Ability to communicate effectively across cyber and physical domains.

- Understanding of how design decisions in the cyber domain may affect the physical domain and visa-versa

- Recognition of the need for, and ability to engage in lifelong learning.

- Knowledge of contemporary issues with cyber-physical systems.

- Ability to select and use appropriate techniques, skills, and modern engineering tools that span the cyber and physical domains.

Most of the ABET criteria were addresses by two-year long ILERT project: International Learning Environment for Real-Time Software Intensive Control Systems (RSIC) started in 2006 [2]. The project was sponsored by the Fund for Improvement of Postsecondary Education (FIPSE) of the U.S. Department of Education and the European Commission. The project was implemented in a consortium 
of one American (ERAU - Embry Riddle Aeronautical University, Daytona Beach, FL) and three European universities (AGH - AGH University of Science and Technology, Krakow, Poland; UJF - Université Joseph Fourier, Grenoble, France; and BUT - Brno University of Technology, Brno, Czech Republic). The academic departments leading the project represented computing, control, and telecommunication disciplines.

Based on own research and the industry surveys, the consortium faculty confirmed that modern computer control systems are heavily software-centric, implementing reactive and time critical software, where safety is the issue and the margin for error is narrow. Examples include aircraft avionics, air traffic control, space shuttle control, medical and automotive equipment, power stations and more. It is vital for future software developers to understand the basic real-time applications concepts such as the issues of timing, concurrency, inter-process communication, resource sharing, interrupts, and handling external physical devices. Generally, challenge is the situation where the physical systems operating in Newtonian (absolute) time must interface with computational systems and networks evolving in cyber time.

The purpose of ILERT project was defined as follows [2]: ... \{four university partners\} propose a study leading to establishment of a RSIC international, multidisciplinary curriculum framework focusing on important aspect of the computer/system/control/software engineering education. We plan to explore the mechanism for involving students from multilingual geographically separated institutions in a coordinated educational experience exposing them to the problems, methods, solution techniques, infrastructure, technologies, regulatory issues, and tools in the domain of dependable real-time safety-critical software-intensive control systems.

The consortium members were deeply convinced that engineering curricula require continuous modifications to prepare students for the technological challenges of the modern workplace. Rapid progress of computing technologies is the major reason that the academic programs like electronics, computer and software engineering, robotics, and control engineering need continuous updates and integration of knowledge.

An additional issue was the internationalization and globalization of complex systems development. Several large companies, specifically in the aerospace industry, engage international teams working in geographically diverse locations often using diverse standards, guidelines, and processes. It is advantageous for future engineers to understand the implications of international collaboration and to appreciate cultural differences.

Successful ILERT project was subsequently continued by Dependable Systems International Research and Education Experience (DeSIRE2) project which started in 2008 [3]. Two American universities joined the consortium but only one continued and contributed to the project (UCF -
University of Central Florida, Orlando, FL). The objective of this implementation project was to establish a platform for a sustained and consistent mobility exchange of students engaged in R-TCPS oriented programs. The target of the programs was to deliver on the labor market graduates, capable of working efficiently in multidisciplinary teams and participating in international collaboration on industrial RTCPS projects, which require conformance to specific standards mandated by regulatory authorities.

This paper summarizes the results of both projects, as an example of a Real-Time Cyber-Physical Systems education framework.

\section{FIRSt PROJeCT: CURRICUlum DESIGN}

The first project (ILERT) started with a research phase, including the analysis of industry requirements related to graduates in the proposed domain. The collected data were analyzed and the results were used to help identify academic program learning objectives and outcomes, thus preparing a base for creation of a new curriculum framework [3].

The subsequent steps included:

- Defining learning objectives and outcomes, identifying the curriculum framework, exploring the partners' programs common features, laboratory infrastructure, identification of the curricula contents, and analysis of the educational process assessment. One needs to note, that the existence of common characteristics did not imply automatic commonality of the ways how individual institutions pursued common educational objectives. Universities often find their own methods to let shared content shape the development of procedure and instructional delivery.

- Defining the roles of the project partners and focus of interest. Considering the four universities strengths in various areas such as control, software engineering, telecommunication, and embedded systems, the consortium defined as their point of interest Real-time Software-intensive Control (RSIC) - a subset of RTTCPS domain.

- Curriculum development included two complementary groups of activities. The first one, more general, had focused on methodology and process of creation of a unique and curriculum framework in the area of real-time software-intensive control, which combines elements of control, software, and computer engineering programs. Such curriculum was in high demand by industry as shown in earlier industry surveys and interviews. The second was a practical case study adapting a selected RSIC curriculum unit acceptable for engineering programs in four organizations. The curricula were reviewed to prioritize and integrate the various elements in order to meet the learning objectives and demands of interdisciplinary specialization in Real-Time SoftwareIntensive Control serving RSIC. An appropriate sequence of courses was proposed. 
- Credit transfer and accreditation issues. The development of new curriculum framework in information technologies may require new approaches to validate and accredit learning. Existing and emerging structures for accreditation, quality control and credit transfer (such as the European Credit Transfer and Accumulation Scheme) were analyzed and coordinated. The proposed curriculum units, focusing on the objectives and outcomes of the educational activity, were developed according to the U.S. Accreditation Board of Engineering and Technology (ABET) standards as well as the applicable standards of Ministry of Higher Education in the European countries.

- Students mobility. Based on the developed curricula, students' mobility between partners institutions was proposed, opening possibility of collaborating and enrolling in the same course offered concurrently in four partner sites.

The project identified six RSIC Framework areas [4]: Software Engineering, Digital Systems, Computer Control, Real-Time Systems, Networking, and Systems Engineering.

1. Software Engineering: software engineering concepts and practices, lifecycle models, project management, processes, software modeling and formal representation; software requirements; software architectural and module design; software construction methods and practices, testing and quality assurance; software maintenance, notations and tools.

2. Digital Systems: methods, techniques, and tools used to support the design of combinational and sequential digital circuits and the design of fault tolerant and advanced networked hardware components.

3. Computer Control: concepts of feedback control, continuous vs. discrete models of dynamic systems, simulation, controller design, implementation of control algorithms in real-time, integrated control design and implementation (hardware-in-the-loop), security aspects, implementation for physical processes.

4. Real-Time Systems: timing and dependability properties of software intensive systems, RTOS concepts and applications, concurrency, synchronization and communication, scheduling, reliability and safety aspects.

5. Networking: data communication, network topology, analysis and design, information security, algorithms, encryption, bus architectures, wireless, distributed control and monitoring.

6. System Engineering: system engineering concepts, principles, and practices; system engineering processes (technical and management), system requirements, design, integration, and testing; special emphasis on the development of a RSIC system and the integration of RSIC system elements.

The basic organizational unit for this framework was RSIC "component". A RSIC component was defined as a curriculum unit covering theory, knowledge, and practice - supporting the RSIC objectives and outcomes. The proposed RSIC Curriculum Framework did not specify the way in which component topics might be formed into modules or courses reflecting the RSIC concept. Component topics were focused in one or two courses, or spread among several courses, along with other non-RSIC topics. For each component more detailed didactic aspects were defined such as: the prerequisite knowledge, component learning objectives, information about required facilities and equipment, and guidelines and suggestions for course design and delivery.

In addition to the above six RSIC Framework areas, additional institutional, regional, or national requirements in areas of "general education" were required to be added to the curricula as, for example, requirements for oral and written communication, for arts and humanities, or for the social sciences. These areas also supported the RSIC curriculum objectives and outcomes related to ethical and professional responsibility, effective communications skills, ability to work as part of a team, and lifelong learning. Each ILERT partner analyzed how the framework could be applied to their program. The challenge was to maintain the program integrity and at the same time include all necessary elements of the RSIC Framework.

\section{SECOND PROJECT: STUDENT EXCHANGES}

Subsequently, another project received support from both FIPSE and European Commission under umbrella of Atlantis Program designed to support trans-Atlantic student mobility exchanges between Europe and USA. The objective of Dependable Systems International Research and Educational Experience project (DeSIRE2) [4] was to train engineering students to achieve global competency and to provide exposure for the students to work in international settings with foreign partners, to get opportunity for exploring the host country language and customs, and to make available instruction the students in the areas not available in the home school.

The learning objectives and outcomes of DeSIRE2 project were to prepare specialists to solve problems in a multidisciplinary way utilizing wide range of CPS domains. The expected graduate student competencies provided by completion of the DeSIRE2 program were as follows:

- Demonstrate professionalism in work and grow professionally through continued learning.

- Demonstrate understanding of analysis and design to implement software-intensive systems.

- Demonstrate understanding of analysis and design to implement control systems.

- Apply advanced software engineering techniques to implement real-time concepts.

- Implement a rigorous quality assurance process.

- Implement hardware/ software integration. 
- Demonstrate knowledge of the principles and techniques needed for the analysis and design of a system form dependability perspective.

- Assume a variety of roles in teams of diverse membership.

According to the initial plans, the student mobility was to be distributed between the original six consortium partner universities: three from the EU in cooperation with three universities from the US. During the first year of the project a multilateral Memorandum of Understanding (MoU) was signed by partners' schools. The memorandum stipulated the conditions of: mobility exchange period, tuition and enrollment, student eligibility, student and faculty mobility, and student selection. Due to legal issues, the third American university left consortium in the first year not contributing to the mobility exchanges.

Each consortium university partners had a dedicated unit in their organizational structure handling foreign students' exchanges and supporting the recruitment. Students received support in both pre- and post-mobility activities. The project contact faculty at each university served as the focal point to advise, guide, and help both local and the visiting students.

Individual program of study was developed for each candidate. The reason was that students from partner universities have had different capabilities and knowledge base due to the differences of their local academic programs. Therefore, it was necessary to shift from courses forcing the "one size fits all" approach to courses accommodating individual learning paths. Such paradigm shift was supported by the "component" approach described in the Section 2.

Due to the individual nature of each exchange and necessity to find appropriate selection of courses, the task of creating an individual learning path for a single exchange was rather complicated. The logistic of the mobility exchange required a candidate student to register for course work at the home institution in coordination with the local mobility exchange project contact faculty, who helped the student to find an appropriate set of courses in one of the overseas partners' schools. The main objective was to focus the individual plan of study in the area related to DeSIRE2 project specialization. The students then took the course overseas, with the grade passed back to the home school international office and recorded with the school registration office (or a dean office) for inclusion in the student's official record. The administrative issues such as course equivalency, satisfying the student's program core requirements, prerequisite requirements etc., were usually approved by the Faculty Dean and appropriate graduate program coordinator in advance before commencement of the mobility exchange. The approved courses were counted towards a degree at the home institution. The mechanism of ECTS/US credits equivalents, as defined by the MoU, was applied.

The consortium partners exchanged 36 students (nineteen from US studying in Europe and seventeen EU students studying in the US) achieving nearly balanced mobility exchanges participation. It needs to be noted that the consortium faced significant challenge recruiting American students. The major obstacles were constraints of academic calendar, missing opportunities of earning while abroad and thus related financial issues, and difficulties with finding appropriate set of courses to be credited to the student transcript [5].

The project requested students to complete post-mobility survey. The sample of students engaged in the mobility is still statistically insignificant, but the post-mobility surveys they filed provide interesting observations. At the project completion we collected total of 30 surveys (83\% return rate): 15 from European students studying in the USA and 15 from the American students studying in Europe.

On a scale of 1 (negative) to 5 (positive), the level of satisfaction related to the program merit, in terms of importance and meeting the goals, are for American students in Europe 3.93 and 4.49 respectively, while for European students in the USA they are 4.13 and 4.40 (Figure 1).

\begin{tabular}{|c|c|c|}
\hline IMPORTANCE & to EU & to US \\
\hline Earning credits toward my degree & 3.9 & 4.3 \\
\hline Earning credits toward my major & 3.6 & 3.9 \\
\hline Acquiring or improving a second language & 3.7 & 4.7 \\
\hline Engaging in a research project & 2.4 & 3.0 \\
\hline Developing personally & 4.2 & 3.7 \\
\hline Gaining new perspectives on my studies & 4.0 & 4.1 \\
\hline Preparing for or advancing my graduate studies & 2.2 & 3.4 \\
\hline Gaining experience for my career & 3.9 & 4.2 \\
\hline Exploring places I have studied at my home school & 3.5 & 3.6 \\
\hline Visiting friends or relatives who live in that region & 3.3 & 1.8 \\
\hline average & 3.47 & 3.68 \\
\hline selected items average & 3.93 & 4.13 \\
\hline MEET MY GOAL & to EU & to US \\
\hline Earning credits toward my degree & 4.5 & 4.7 \\
\hline Earning credits toward my major & 4.4 & 4.5 \\
\hline Acquiring or improving a second language & 4.0 & 4.3 \\
\hline Engaging in a research project & 3.6 & 2.5 \\
\hline Developing personally & 4.7 & 4.1 \\
\hline Gaining new perspectives on my studies & 4.5 & 4.5 \\
\hline Preparing for or advancing my graduate studies & 3.6 & 3.5 \\
\hline Gaining experience for my career & 4.4 & 4.4 \\
\hline Exploring places I have studied at my home school & 3.9 & 3.9 \\
\hline Visiting friends or relatives who live in that region & 3.6 & 2.4 \\
\hline average & 4.11 & 3.87 \\
\hline selected items average & 4.49 & 4.40 \\
\hline
\end{tabular}

Fig.1. DeSIRE mobility exchanges: importance and meeting goals 
The satisfaction survey results show that the overall value of the program was nearly perfect 4.93 for European students visiting USA and 4.57 for Americans in Europe. The averages of the relevant survey items are: 4.10 for the European students in USA and slightly lower 3.74 for the Americans in Europe (Figure 2). Statistical t-test analysis allows us to state with $88 \%$ confidence that the experiences of European students in USA seem to be more positive that their counterpart in Europe.

\begin{tabular}{|l|c|c|}
\hline & to EU & to US \\
\hline Overall value of the program & 4.57 & 4.93 \\
\hline Overall organization of this program & 3.4 & 4.5 \\
\hline Assistance provided by host institution & 3.7 & 4.7 \\
\hline Availability of host coordinator & 4.1 & 4.7 \\
\hline On-site first contacts and orientation & 3.7 & 4.6 \\
\hline Arrival arrangements by host institution & 2.8 & 4.6 \\
\hline Access to computing labs/internet & 2.5 & 4.6 \\
\hline Engagement in research and project activities & 3.5 & 3.1 \\
\hline Interactions with students from the host school & 3.7 & 3.8 \\
\hline Interactions with faculty from the host school & 3.7 & 4.2 \\
\hline Organizational assistance at the host institution & 3.0 & 4.4 \\
\hline Opportunity to improve foreign language skills & 4.0 & 4.7 \\
\hline Accessibility of public transportation & 4.8 & 1.5 \\
\hline General comfort at your housing & 3.7 & 4.1 \\
\hline Housing proximity to the university & 3.7 & 4.7 \\
\hline Security and Safety on Campus & 4.1 & 4.9 \\
\hline Cost in comparison to other housing & 4.5 & 3.2 \\
\hline Availability of university meal services & 4.5 & 4.1 \\
\hline Quality of the meals & 3.9 & 3.3 \\
\hline
\end{tabular}

Fig.2. DeSIRE mobility exchanges: satisfaction survey

Due to the demanding nature of engineering courses conducted in English in three European partner universities, the issue of the language instruction was not a priority. However, the exchange students engaged in language and/or cultural instruction classes, participated in a variety of activities to learn the host country, interacted with their peers from the host school, and in general were satisfied with the opportunity to travel and explore different cultures. The native Slavic languages in two European partner universities were rather difficult to learn for the American students - except those with the specific country heritage that may already know the language (being typically an additional initiative to apply for the mobility exchange)

Additionally, faculty mobility exchanges were conducted to support: lectures delivery at the host institutions related to the objectives of the project, pre-mobility orientations and program promotion at host institutions, face-to-face meetings with mobility candidates and active mobility students, and planning/co-ordination meetings with the host university faculty and administration.

A dedicated website supporting the student exchange was maintained and systematically upgraded, containing up-to date information about the project objectives, activities, outcomes, progress and deliverables. One of the requirements of the mobility exchange completion was for the students to create personal blogs reflecting their experiences. The blogs were linked from the project website (http://www.desire.agh.edu.pl/).

All students engaged in the program had very positive impression of their mobility stay and appreciated the opportunity provided by the project funding. In general the students expressed positive feedback related to the general study abroad experience. Only few comments from postmobility surveys addressed the actual classroom experience.

- To Europe:

- ...academic system is a little harder than US because of the lack of assignments and the dominant influence of the exams (midterm and final) on your grade for the class (ERAU->UJF).

- ...enjoyed very much the curriculum and the hands on approach of the courses, especially the opportunity to practice our newly gained knowledge through the academic project. I believe that this experience thought me a lot on more than just academic knowledge but it also allowed me to grow on a more personal level (UCF->UJF).

\section{- To USA:}

- ...engineering and software departments are also very good here. There is lot of interesting projects, classes. Another great thing is that the teachers are mostly people that are working or worked in the field they are now teaching, i.e. I had Software Project Management class with the instructor from Boeing Company - a real software project manager (AGH->ERAU)

-... this was a real experience for me abroad. It brought me a lot of knowledge in certain class and a new method of working (UJF->ERAU).

\section{IMPLEMENTATION EXAMPLES}

Hands-on learning approach was proposed for the majority of RSIC components. This approach involves learning experience which enhances student's ability to think critically. The student must plan an experiment to test a hypothesis, put the experiment into motion, see the experiment to completion, and then be able to explain and interpret the experiment's results. Another hands-on experience has been engagement in a team project requiring students to analyze the problem, specify system requirements, find appropriate solution, resulting in designing and implementing a small scale cyber-physical system. Two selected examples are provided below.

\section{A. Example 1: CPS Laboratory Experiment}

The example of lab environment supporting education in Real-Time Cyber-Physical Systems is provided below [6].

The goal of this educational module (lectures and practical training) is to familiarize students with industrial Ethernet IP technology studying a model of an industrial Allen Bradley Networked Programmable Logic Controller (PLC) connected through different gateways to the physical system (model of air lift). The system uses distance 
measurements and implements control signals in a similar way to typical industrial process. Students became familiar with essential characteristics of data transmission networks and with the influence of the network parameters on control quality of the physical system. Students also work with several standard testing methods and items of industrial equipment.

The Ethernet IP laboratory, shown in Figure 4 consists of six data transmission nodes: CompactLogix L35E Programmable Logic Controller, POINT_IO: 1734-AENT, PowerFlex 40 inverter, WAGO 750-341 Coupler, Internet Camera (WebCam), and PanelView 600 Plus - Touch Panel.

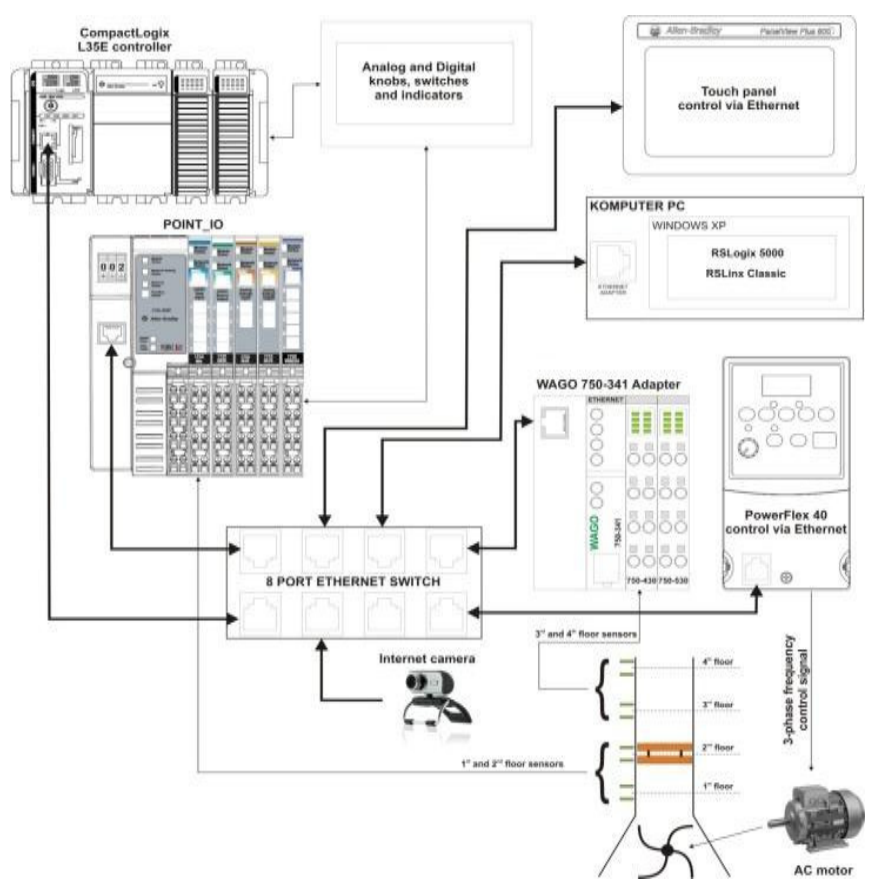

Fig.3. Laboratory Setup

A standard personal computer is used as a development and Ethernet monitoring platform and the web camera generates noise imitating the network data traffic.

The major issues for consideration by the students were: (a) analyze the Ethernet/IP packets and Ethernet network parameters (throughput, round trip time etc.) by utilizing the Wireshark application, (b) diagnose and analyze the jitter for a several scan times and the RPI parameter (Request Packet Interval), and (c) find the effects of load Ethernet network on stabilization of the position of the Aero-lift.

The physical system used in the module is Aero-lift. The test-rig contains the blower based on three-phase drive controlled by the inverter, a pipe for vertical motion of the cart and eight discrete sensors to measure the cart position (see Figure 4). The Aero-lift is an example of the dynamic system sensitive for sampling time and latency in data transmission. With this set-up a number of consequences caused by the inaccurate settings for Ethernet modules and I/O blocks can be observed.

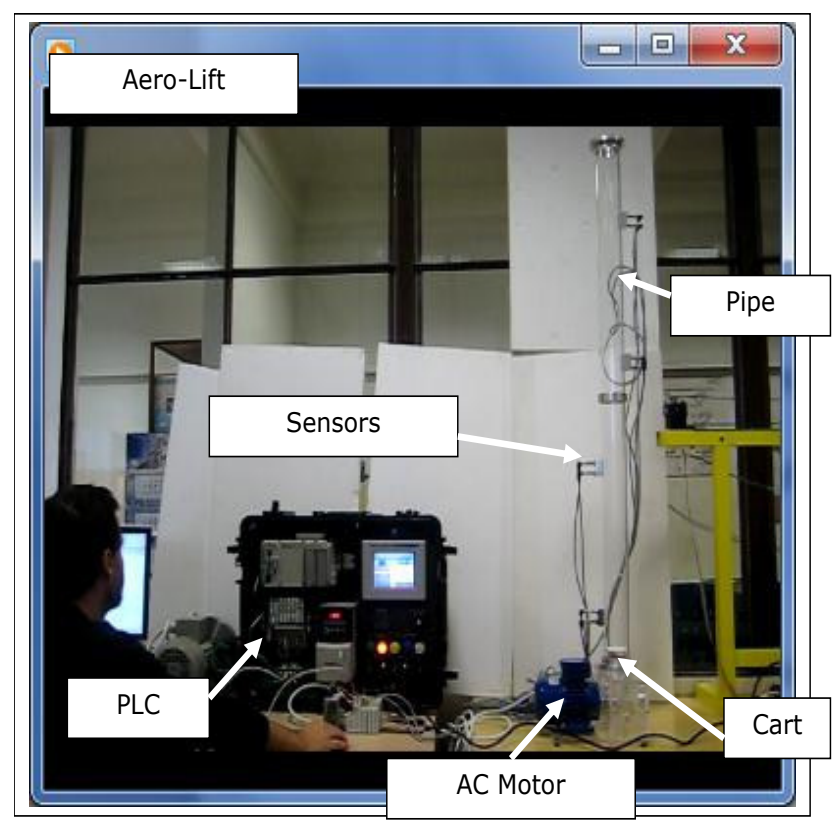

Fig.4. Physical system used in the laboratory setup

The offered laboratory demonstrates all the features and includes all components expected for Cyber Physical Systems: industrial equipment reflecting real physical plant, computing environment, time critical software, and distance data transfer. The laboratory shows that traditional real-time performance guarantees are insufficient for CPS when system components are spatially distributed and connected via data transmission networks.

\section{B. Example 2: Teamwork Development of a CPS}

The consortium members recognized the demand for efficient development of quality RSIC/ CPS systems and need for an international learning environment. An innovative learning approach was implemented involving students from multilingual, geographically separated institutions in a coordinated educational experience in laboratory CPS development [7]. The project was realized as a component of the ILERT engaging on-site students and faculty of universities from four countries (USA, Poland, France, and Czech Republic).

The technical objective of the project was to develop realtime software packages for two robots (physical system) that cooperate in searching for a target in a simple maze. The target search in the maze was designed to simulate a practical example a rescue problem.

The two robots were considered to work within the limited operating area. The robots were assigned different roles: The "Leader" Robot starts in one of the four corners and, according the implemented algorithm, it is searching for the black square that is positioned in the center of the maze. The "Follower" Robot starts in one of the four corners (which may be different that the starting corner of the 
Leader Robot) and according the information received from the Leader robot it attempts to find the black corner is a shortest possible time. The use of rigorous software development process was emphasized and the comprehensive documentation was expected as an integral part of the project's outcomes. Team cooperation and related intensive inter- and intra-team communication was also assumed. Each team was responsible for their developed components from the initial phases of analysis and design through component implementation to integration and testing.

The two major Project Learning Objectives were focusing on the Process and Product:

- Process - effective work in multicultural and multidisciplinary teams including such aspects as team organization and planning, team communication, and project management.

- Product - development of an RSIC system resulting in developing real-time embedded software, hardware and software system integration, implementation of digital control in real time, design/implement communication protocols.

To meet the above objectives, international students' teams have worked on robotic design and control experiments with LEGO MINDSTORMS NXT kits.

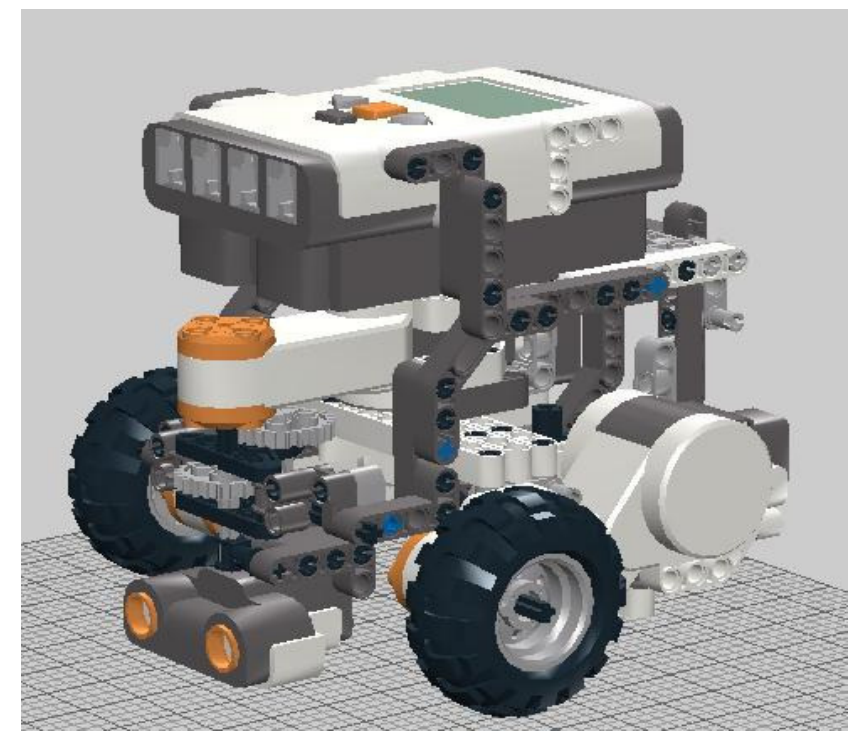

Fig.5. Design of three wheels robot construction

The basic problem was an accurate and precise motor control. The error in the motor control can affect the correct navigation and robot movements. From the educational viewpoint it was not so important that the robot moves, but how precise it operated and what was the accuracy of the controlled devices.

LEGO-MINDSTROMS NXT kit (Fig. 5) was selected as a common platform for students' teams. The used LEGO platform proved flexible enough to achieve both technical learning objectives. A Web-based Project Management
System (WebPMS) was used to improve communication and test Internet based tools for inter-university collaboration.

The programming solutions related to LEGO NXT were implemented in open source NXC (Not Exactly C) - a textbased language for MINDSTORMS® NXT robot microcontroller. The language supports multitasking on standard LEGO firmware. With freely available BrixCC Integrated Development Environment (IDE) it was possible to create quite complex and advanced data acquisition and control algorithms operating concurrently and in real-time, including file management and communication features. A custom Bluetooth communication program was designed and implemented so the $\operatorname{robot}(\mathrm{s})$ movements could be monitored and displayed on the host.

\section{CONCLUSION}

Cyber-Physical Systems are a novel category of engineering systems, involving closely interacting physical and computational components. In the CPS, control algorithms, embedded hardware, real-time software, and communication are closely intertwined while the digital cyber components are connected interacting with the physical real-world processes. Due to interaction with the environment and people, the issues of dependability and performance while meeting the functional requirements are of primary concern. It is critical that the future CPS developers are proficient in these multidisciplinary projects while being able to work with professionals representing different skills and organizational affiliations.

The laboratory environment delivers required features and includes all components expected for Cyber Physical Systems: physical plant, computing environment, software intensive and time critical, distance data transition. CPS technology challenges included: CPS data acquisition, data precision, time dependencies, the critical elements of modern CPS, quality assurance and integration; data and information analytics, control algorithms and rigorous software development process. In particular, assures compliance with an important CPS education criterion formulated by ABET: "ability to function effectively on multi-disciplinary teams spanning cyber and physical domains".

The international consortium of universities, with support of the U.S. Department of Education FIPSE and the European Commission projects, has been engaged in the CPS related educational activities at graduate levels since 2007. The objective of these educational activities was to educate students in the principles and design issues in CPS and to develop student competency to be comfortable working in an international setting collaborating across the nations' boundaries. It was found that it has been easier to achieve these multi-disciplinary and integration-focused knowledge and skills required for the CPS education when engaging a group of universities, rather than when attempting to achieve them within a single university. Students' feedback confirms that they truly enjoyed the 
program, and they expressed great interest in future participation in the CPS related activities.

To continue potential future cooperation after project completion and expiration of the multilateral agreement, the participating universities signed bi-lateral agreements stipulating continuing cooperation in the area of the CPS, with both faculty and student exchanges.

\section{REFERENCES}

[1] ABET Engineering Criteria Program Educational Outcomes, available http://www.foundationcoalition.org/home/keycomponents/assessment _eval/ec_outcomes_summaries.html, [Accessed March 15, 2015].

[2] W. Grega, A. Kornecki, M. Sveda, and J. Thiriet, "Developing Interdisciplinary and Multinational Software Engineering Curriculum", in Proceedings of the ICEE '07, Coimbra, Portugal, Sep. 3-7, 2007 http://www.desire.agh.edu.pl , [Accessed March 15, 2015].
[3] A.J. Kornecki, W. Grega,, T. Hilburn, J-M. Thiriet, M. Sveda, O. Rysavy, A. Pilat,“Transatlantic Engineering Programs: An Experience in International Cooperation", "Engineering the Computer Science and IT", Chapter 5, In-Tech Publishing, Croatia, 2009, ISBN 978-953307-012-4, pp. 65-84.

[4] M. Sveda, W. Grega, T. Hilburn, A. Kornecki, O. Rysavy, J-M. Thiriet, (2009), "Real-Time Software-Intensive Systems Engineering in an International Perspective", in Proceedings of 20th EAEEIE Annual Conference, Valencia, Spain, June 22-24, 2009.

[5] A.J. Kornecki, W. Grega, A. Gonzalez, "Europe/USA Mobility Exchange in Information Engineering: Why Is It Less Attractive to the American Students?", in Proceedings of EIEAEE, June 2010 Palanga, Lithuania.

[6] CoNeT Mobile Lab, Ethernet IP - Section 5, CML User Manual, Rev. 1.0, http://www.ipnet.agh.edu.pl/Materials2/Module5/Module5UserManual.pdf, [Accessed March15, 2015].

[7] A. Pilat, A.J. Kornecki, J-M. Thiriet, W. Grega, O. Rysavy , (2009), "Inter-university Project Based on LEGO NXT" , in Proceedings of 3rd IEEE Multi-conference on Systems and Control, Saint Petersburg, Russia, July 8-10, 2009, ISBN: 978-1-4244-4602-5, ISSN: 1085-1992. 